\title{
Generating function for nucleus-nucleus scattering amplitudes in Glauber theory
}

\author{
Yu. M. Shabelski* and A. G. Shuvaev $\oplus^{\dagger}$ \\ Petersburg Nuclear Physics Institute, Kurchatov National Research Center, Gatchina, Saint Petersburg 188300, Russia
}

(Received 17 June 2021; revised 1 November 2021; accepted 24 November 2021; published 9 December 2021)

\begin{abstract}
A new approach to deal with the scattering amplitudes in Glauber theory is proposed. It relies on the use of a generating function, which has been explicitly found. The method is applied to the analytical calculation of the nucleus-nucleus elastic scattering amplitudes in the all interaction orders of the Glauber theory.
\end{abstract}

DOI: 10.1103/PhysRevC.104.064607

\section{INTRODUCTION}

The theory of nucleus-nucleus interaction is an important aspect of a long-standing multiple-scattering problem. It has acquired a modern impetus from the large number of the currently available experimental data (see, e.g., Refs. [1-4]). The theoretical calculations provide, in particular, a way to get information on scattering of both stable and unstable nuclei at the comparatively high energies of more than several hundreds of $\mathrm{MeV}$ per nucleon. The calculations are standardly carried out in the Glauber approach [5,6]. It has proven to be highly efficient for the hadron-nucleus collision, supplying rather simple analytical expressions for the scattering amplitudes. The case of the nucleus-nucleus scattering is much more involved. Additional simplifying approximations are commonly used to obtain an analytical expression such as the optical model or the rigid target model (see, e.g., Refs. [7-9]). Apart from these models there are only numerical calculations based on the Monte Carlo method or on its modifications [10-13].

In the present paper we propose a novel approach. Assuming the range of nucleon-nucleon interaction to be small compared to the typical nucleus size we have derived the analytical expression for the generating function giving the Glauber amplitudes for nucleus-nucleus scattering. Its relatively simple form allows one to reach the same accuracy as that provided with the numerical Monte Carlo calculations without being as time-consuming as they are.

\section{SCATTERING AMPLITUDES IN THE GLAUBER THEORY}

To begin, we briefly outline the basics of the Glauber theory. The amplitude of the elastic scattering of the incident

\footnotetext{
*shabelsk@thd.pnpi.spb.ru

†shuvaev@thd.pnpi.spb.ru
}

Published by the American Physical Society under the terms of the Creative Commons Attribution 4.0 International license. Further distribution of this work must maintain attribution to the author(s) and the published article's title, journal citation, and DOI. Funded by $S C O A P^{3}$. nucleus $A$ on the fixed target nucleus $B$ reads $[14,15]$

$$
F_{A B}^{\mathrm{el}}(q)=\frac{i k}{2 \pi} \int d^{2} b e^{i q b}\left[1-S_{A B}(b)\right],
$$

where $q$ is the transferred momentum and $k$ is the mean momentum carried by a nucleon in nucleus $A$. The twodimensional impact momentum $b$ lies in the transverse plain to the vector $k$. The main assumption underlying the Glauber theory is that the radius of the nucleon-nucleon interaction is much smaller than the typical nucleus size. Then assuming the phase shifts of the nuclear scattering to be the sum of those for each nucleon-nucleon scattering, $\chi_{N N}(b)$, the function $S_{A B}(b)$ takes the form

$$
S_{A B}(b)=\left\langle A, B\left|\left\{\prod_{i j}\left[1-\Gamma_{N N}\left(b+x_{i}-y_{j}\right)\right]\right\}\right| A, B\right\rangle,
$$

with

$$
\Gamma_{N N}(b)=1-e^{i \chi_{N N}(b)}=\frac{1}{2 \pi i k} \int d^{2} q e^{-i q b} f_{N N}^{\mathrm{el}}(q),
$$

where $f_{N N}^{\mathrm{el}}(q)$ is the nucleon-nucleon scattering amplitude. The product in Eq. (2) comprises all pairwise interactions between the nucleons from the projectile and target nuclei $A$ and $B$, with symbol $\langle A, B|\cdots| A, B\rangle$ standing for an average over the nucleons' positions $x_{i}$ and $y_{j}$ in the transverse plain. Each pair $\{i, j\}$ enters the product only once, meaning that each nucleon from the projectile nucleus can scatter on each nucleon on the target once but no more than once.

The total interaction cross section is

$$
\sigma_{A B}^{\mathrm{tot}}=\frac{4 \pi}{k} \operatorname{Im} F_{A B}^{\mathrm{el}}(q=0)=\int d^{2} b\left[1-S_{A B}(b)\right],
$$

while the integrated elastic cross section is

$$
\sigma_{A B}^{\mathrm{el}}=\int d^{2} b\left[1-S_{A B}(b)\right]^{2} .
$$

The difference between these two values determines the total inelastic, or reaction, cross section,

$$
\sigma_{A B}^{r}=\sigma_{A B}^{\mathrm{tot}}-\sigma_{A B}^{\mathrm{el}}=\int d^{2} b\left[1-\left|S_{A B}(b)\right|^{2}\right] .
$$




\section{GENERATING FUNCTION}

A main obstacle to dealing with the Glauber amplitude (2) is its complicated combinatorial structure. To treat it analytically we first rewrite it more explicitly through nucleon distributions in the colliding nuclei,

$$
\begin{aligned}
S_{A B}(b)= & \int \prod_{i=1}^{A} d^{2} x_{i} \int \prod_{j=1}^{B} d^{2} y_{j} \rho_{A}^{\perp}\left(x_{1}-b, \ldots, x_{A}-b\right) \\
& \times \rho_{B}^{\perp}\left(y_{1}, \ldots, y_{B}\right) \\
& \times\left\{\prod_{i j}\left[1+g \Gamma_{N N}\left(x_{i}-y_{j}\right)\right]\right\}
\end{aligned}
$$

Here the nucleon densities in the transverse plain, $\rho_{A, B}^{\perp}$, are determined through three-dimensional ones integrated over longitudinal coordinates,

$$
\begin{aligned}
\rho_{N}^{\perp}\left(x_{1}, \ldots, x_{N}\right)= & \int \prod_{i=1}^{N} d z_{i} \rho_{N}\left(z_{1}, x_{1}, \ldots, z_{N}, x_{N}\right), \\
& \times \int \prod_{i=1}^{N} d^{3} r_{i} \rho_{N}\left(r_{1}, \ldots, r_{N}\right)=1 .
\end{aligned}
$$

For later convenience an extra parameter $g$ counting the number of interactions is introduced in Eq. (7), really $g=-1$. We also assume in what follows that the three-dimensional nuclear densities are reduced to the product of one-nucleon densities,

$$
\rho_{N}\left(r_{1}, \ldots, r_{N}\right)=\prod_{i=1}^{N} \rho_{N}\left(r_{i}\right), \quad \int d^{3} r \rho_{N}(r)=1,
$$

and consequently

$$
\rho_{N}^{\perp}\left(x_{1}, \ldots, x_{N}\right)=\prod_{i=1}^{N} \rho_{N}^{\perp}\left(x_{i}\right), \quad \int d^{2} x \rho_{N}^{\perp}(x)=1 .
$$

This assumption means the nucleon-nucleon correlations are neglected.

The next step is to represent Eq. (7) as a functional integral. To this end let us consider the identity

$$
\begin{aligned}
C_{0} \int & D \Phi D \Phi^{*} \exp \left\{-\int d^{2} x d^{2} y \Phi(x) \Delta^{-1}(x-y) \Phi^{*}(y)\right. \\
& \left.+\sum_{i} \Phi\left(x_{i}\right)+\sum_{j} \Phi^{*}\left(y_{j}\right)\right\} \\
= & \exp \left\{\sum_{i, j} \Delta\left(x_{i}-y_{j}\right)\right\}=\prod_{i, j} e^{\Delta\left(x_{i}-y_{j}\right)}
\end{aligned}
$$

where $C_{0}$ is the normalization constant and the functional integral can be thought of as an infinite product of twodimensional integrals over the auxiliary fields $\Phi(x)$ at each space point $x$. The inverse of the propagator, $\Delta^{-1}(x-y)$, is understood in a functional sense, $\int d^{2} z \Delta^{-1}(x-z) \Delta(z-$ $y)=\delta^{(2)}(x-y)$. If this function is chosen to obey the equation

$$
e^{\Delta(x-y)}-1=g \Gamma_{N N}(x-y),
$$

the right-hand side of Eq. (9) recovers the product in Eq. (7). The function $\Delta(x-y)$ plays a role similar to that of the Mayer propagator (function) in statistical mechanics; the analogy between Glauber theory and statistical mechanics has been remarked on earlier (see, e.g., Ref. [16]). Then we get

$$
\begin{aligned}
& S_{A B}(b) \\
& =C_{0} \int D \Phi D \Phi^{*} \exp \left\{-\int d^{2} x d^{2} y \Phi(x) \Delta^{-1}(x-y) \Phi^{*}(y)\right\} \\
& \times\left[\int d^{2} x \rho_{A}^{\perp}(x-b) e^{\Phi(x)}\right]^{A}\left[\int d^{2} y \rho_{B}^{\perp}(y) e^{\Phi^{*}(y)}\right]^{B} \cdot(11)
\end{aligned}
$$

This form suggests that it is natural to introduce the generating function

$$
\begin{aligned}
Z(u, v)= & \int D \Phi D \Phi^{*} \exp \left\{-\int d^{2} x d^{2} y \Phi(x) \Delta^{-1}(x-y) \Phi^{*}(y)\right. \\
& \left.+u \int d^{2} x \rho_{\perp}(x-b) e^{\Phi(x)}+v \int d^{2} x \rho_{\perp}(x) e^{\Phi^{*}(x)}\right\},
\end{aligned}
$$

so that

$$
S_{A B}(b)=\left.\frac{1}{Z(0,0)} \frac{\partial^{A}}{\partial u^{A}} \frac{\partial^{B}}{\partial v^{B}} Z(u, v)\right|_{u=v=0} .
$$

The generating function (12) is the focus of the present paper. Being analytically evaluated it comprises all interaction orders of the Glauber theory for nucleus-nucleus collision.

\section{EXPLICIT EVALUATION OF THE GENERATING FUNCTION}

As it has been mentioned above the Glauber theory essentially relies on the short-distance nature of the nucleonnucleon interaction. The same property, the small interaction range, makes the complex functional integral (12) feasible. The standard parametrization of the elastic nucleon-nucleon scattering amplitude is

$$
f_{N N}^{\mathrm{el}}(q)=i k \frac{\sigma_{N N}^{\mathrm{tot}}}{4 \pi} e^{-\frac{1}{2} \beta q^{2}}
$$

where $\sigma_{N N}^{\text {tot }}$ is the total nucleon-nucleon cross section. It gives according to Eq. (3)

$$
\Gamma_{N N}(x)=\frac{\sigma_{N N}^{\text {tot }}}{4 \pi \beta} e^{-\frac{x^{2}}{2 \beta}},
$$

the value $a=\sqrt{2 \pi \beta}$ being of the order of the interaction radius. Assuming $a$ to be small at the nuclear scale the nucleon-nucleon amplitude can be treated as a pointlike function,

$$
\Gamma_{N N}(x) \simeq \frac{1}{2} \sigma_{N N}^{\text {tot }} \delta^{(2)}(x)
$$

If $\Delta(x-y)$ is pointlike the integrals over the $\Phi(x)$ fields in Eq. (12) are independent for different coordinate values. This turns the functional integral into the infinite product of 
finite-dimension integrals, which can be separately evaluated for each $x$.

To do this accurately we replace the continuous integrals in the exponent by discretized sums. The discrete version of the identity (9) reads

$$
\begin{aligned}
C_{0} \prod_{x_{n}} \int \frac{d \Phi\left(x_{n}\right) d \Phi^{*}\left(x_{n}\right)}{2 \pi} \exp \left\{-\sum_{n} \frac{1}{y} \Phi\left(x_{n}\right) \Phi^{*}\left(x_{n}\right)\right. \\
\left.\quad+\sum_{i} \Phi\left(x_{i}\right)+\sum_{j} \Phi^{*}\left(y_{j}\right)\right\} \\
=\exp \left\{y \sum_{i, j} \delta_{x_{i}, y_{j}}\right\}
\end{aligned}
$$

where $\delta_{x_{i}, y_{j}}$ is the Kronecker symbol for the discrete nucleons' coordinates, $\delta_{x_{i}, y_{j}} / a^{2} \rightarrow \delta^{(2)}\left(x_{i}-y_{j}\right)$ for $a \rightarrow 0$. Since

$$
e^{y \delta_{x_{i}, y_{j}}}=1+\left(e^{y}-1\right) \delta_{x_{i}, y_{j}}
$$

the right-hand side of the identity (17) yields

$$
\prod_{i, j}\left[1+g \frac{1}{2} \frac{\sigma_{N N}^{\mathrm{tot}}}{a^{2}} \delta_{x_{i}, y_{j}}\right] \rightarrow \prod_{i, j}\left[1+g \Gamma_{N N}\left(x_{i}-y_{j}\right)\right]
$$

whereas Eq. (10) translates into

$$
e^{y}-1=g \frac{1}{2} \frac{\sigma_{N N}^{\text {tot }}}{a^{2}} .
$$

The generating function becomes the product of independent integrals at the points $x_{i}$,

$$
Z(u, v)=\prod_{x_{i}} \int \frac{d \Phi\left(x_{i}\right) d \Phi^{*}\left(x_{i}\right)}{2 \pi} \exp \left\{-\frac{1}{y} \Phi\left(x_{i}\right) \Phi^{*}\left(x_{i}\right)+u a^{2} \rho_{A}^{\perp}\left(x_{i}-b\right) e^{\Phi\left(x_{i}\right)}+v a^{2} \rho_{B}^{\perp}\left(x_{i}\right) e^{\Phi^{*}\left(x_{i}\right)}\right\},
$$

or, after evaluating $\Phi(x)$ integrals in Eq. (19),

$$
Z(u, v)=\exp \left\{\sum_{x_{i}} \ln \left(y \sum_{M, N \geqslant 0} \frac{e^{y M N}}{M ! N !}\left[a^{2} u \rho_{A}^{\perp}\left(x_{i}-b\right)\right]^{M}\left[a^{2} v \rho_{B}^{\perp}\left(x_{i}\right)\right]^{N}\right)\right\} .
$$

The densities are slowly varying at the size $a$, which allows one to replace the sum over $x_{i}$ with the integral,

$$
\begin{gathered}
Z(u, v)=C e^{W_{y}(u, v),} \\
W_{y}(u, v)=\frac{1}{a^{2}} \int d^{2} x \ln \left(\sum_{M \leqslant A, N \leqslant B} \frac{z_{y}^{M N}}{M ! N !}\left[a^{2} u \rho_{A}(x-b)\right]^{M}\left[a^{2} v \rho_{B}(x)\right]^{N}\right),
\end{gathered}
$$

with $u$ - and $v$-independent constant $C$ being irrelevant in Eq. (13) and

$$
z y=1+g \frac{1}{2} \frac{\sigma_{N N}^{\text {tot }}}{a^{2}}
$$

The sums over $M$ and $N$ can always be truncated up to $A$ and $B$ because the higher terms obviously do not contribute to the derivatives in Eq. (13). Put differently, the number of contributions to the generating function does not exceed the number of various brackets in the initial product (2).

\section{RELATION TO THE KNOWN APPROXIMATIONS}

The formulas (20) and (21) are the net result for the generating function. To elaborate it further we expand $W_{y}(u, v)$ into the series built of the densities overlaps,

$$
t_{m, n}(b)=\frac{1}{a^{2}} \int d^{2} x\left[a^{2} \rho_{A}^{\perp}(x-b)\right]^{m}\left[a^{2} \rho_{B}^{\perp}(x)\right]^{n} .
$$

Since $t_{0,1}(b)=t_{1,0}(b)=1$, we have $W_{y}(u, v)=u+v+$ $F(u, v)$ and the amplitude reads

$$
S_{A B}(b)=\left.\sum_{k, j \leqslant A, B} \frac{A ! B !}{(A-k) !(B-j) !} \frac{1}{k !} \frac{\partial^{k}}{\partial u^{k}} \frac{1}{j !} \frac{\partial^{j}}{\partial v^{j}} e^{F(u, v)}\right|_{u=v=0} .
$$

For $A, B \gg 1$ one may assume that $k, j \ll A, B$ and $A ! /(A-$ $k) ! \cdot B ! /(B-j) ! \approx A^{k} \cdot B^{j}$, which gives

$$
S_{A B}(b) \approx e^{F(A, B)}
$$

Really the functions $t_{m, n}(b)$ decrease as the indices $m$ and $n$ grow. Keeping only the lowest, $m=n=1$, we arrive at the well-known optical approximation [7]

$$
F(A, B)=-\frac{1}{2} \sigma_{N N}^{\text {tot }} T_{A B}(b), \quad T_{A B}(b)=A B t_{1,1}(b) .
$$

The optical approximation is equivalent to the requirement that each nucleon from one nucleus interacts with another nucleus no more than once.

Another known approximation easily reproduced here is the rigid target (or projectile) approximation [8,9]. It allows any nucleon from the projectile to interact with several nucleons from the target, whereas any target nucleon can interact no more than once. Though it seems to be rather natural when the atomic weight of the projectile is significantly smaller than that of the target nucleus, this approximation works fairly good even for the equal atomic weights [15]. It requires one density, say, $\rho_{A}^{\perp}(x)$, to be kept in the formula (20) only in the linear order, permitting at the same time any powers of $\rho_{B}^{\perp}(x)$. 
It gives

$$
\begin{aligned}
W_{y}(u, v) & =\frac{1}{a^{2}} \int d^{2} x \ln \left(\sum_{N} \frac{1}{N !}\left[a^{2} v \rho_{B}^{\perp}(x)\right]^{N}+\left[a^{2} \rho_{A}^{\perp}(x-b)\right] \sum_{N} \frac{z_{y}^{N}}{N !}\left[a^{2} v \rho_{B}^{\perp}(x)\right]^{N}\right) \\
& =v+u \int d^{2} x \rho_{A}^{\perp}(x-b) e^{a^{2} v(z y-1) \rho_{B}^{\perp}(x)},
\end{aligned}
$$

yielding the generating function

$$
Z(u, v)=e^{v+u T_{\mathrm{rg}}(v, b)}, \quad T_{\mathrm{rg}}(v, b)=\sum_{n=0}^{\infty} \frac{1}{n !} t_{1, n}(b) v^{n},
$$

which produce for $B \gg 1$

$$
S_{A B}(b)=\left[T_{\mathrm{rg}}(b)\right]^{A}, \quad T_{\mathrm{rg}}(b)=\int d^{2} x \rho_{A}^{\perp}(x-b) e^{-\frac{1}{2} \sigma_{N N}^{\text {tot }} \rho_{B}^{\frac{1}{B}}(x)}
$$

\section{RESULTS FOR ${ }^{12} \mathrm{C}^{-12} \mathrm{C}$ SCATTERING}

Below we present the results obtained with the full generating function (20) for the ${ }^{12} \mathrm{C}^{-12} \mathrm{C}$ scattering in the energy interval $800-1000 \mathrm{MeV}$ per projectile nucleon, where the experimental data exist [17]. The total cross section $\sigma_{N N}^{\text {tot }}=$ $43 \mathrm{mb}$ has been taken from averaging over $p p$ and $p n$ values, and the slope value has been chosen to be $\beta=0.2 \mathrm{fm}^{2}$. The nucleon density is parametrized by harmonic oscillator distribution well suited for light nuclei with the atomic weight $A \leqslant 20$,

$$
\rho_{A}(r)=\rho_{0}\left[1+\frac{1}{6}(A-4) \frac{r^{2}}{\lambda^{2}}\right] e^{-\frac{r^{2}}{\lambda^{2}}}
$$

with $\rho_{0}$ being the normalization, and the factor $\lambda$ being adjusted to match the nuclear mean square radius, $R_{\mathrm{rms}}=\sqrt{r_{A}^{2}}$, where $r_{A}^{2}=\int d^{3} r r^{2} \rho_{A}(r)$.

Upon evaluating $W_{y}(u, v)$ through all $t_{m n}(b)$ functions (22) for $m, n \leqslant A=12$ in the parametrization (27) with $R_{\mathrm{rms}}=$ $2.49 \mathrm{fm}$ fitted for this parametrization in Ref. [13] from Monte Carlo simulation of a ${ }^{12} \mathrm{C}^{12} \mathrm{C}$ collision, we have calculated the reaction cross section (6) and the total cross section (4). Table I compares their values obtained in the optical approximation (25), in the rigid target approximation (26), and with the full generating function for two cases, first assuming $A \gg 1$ and using the approximate formula (24) and second by exact

TABLE I. The reaction and the total cross sections of the ${ }^{12} \mathrm{C}-{ }^{12} \mathrm{C}$ collision at the energy $950 \mathrm{MeV}$ per nucleon and $R_{\mathrm{rms}}=2.49 \mathrm{fm}$. The first two columns present the results of the optical and rigid target approximations, and the second two columns present the results obtained with the full generating function, assuming $A \gg 1$ (third column) and exactly differentiating it (fourth column).

\begin{tabular}{lcccc}
\hline \hline & $\begin{array}{c}\text { Optical } \\
\text { approximation }\end{array}$ & $\begin{array}{c}\text { Rigid target } \\
\text { approximation }\end{array}$ & $\begin{array}{c}\text { Assuming } \\
A \gg 1\end{array}$ & $\begin{array}{c}\text { Exact } \\
\text { differentiating }\end{array}$ \\
\hline$\sigma^{r}(\mathrm{mb})$ & 952 & 911 & 857 & 867 \\
$\sigma^{\text {tot }}(\mathrm{mb})$ & 1572 & 1470 & 1371 & 1363 \\
\hline \hline
\end{tabular}

differentiating the generating function. The last two numbers in the upper row of the table are in reasonable agreement with the experimental value $853 \pm 6 \mathrm{mb}$ [17]. One should bear in mind that the experimentally measured value actually refers to the so-called interaction cross section rather than to the reaction one. The difference between them can be at the several percent level [18]. At the same time the obtained values are close to those of the Monte Carlo calculations with the same parameters and the density parametrization [13].

A word of caution with respect to the formula (24) is needed here. When the generating function is exactly differentiated in Eq. (23), the terms with $m>A$ and $n>B$ in the function $F(u, v)=\sum_{m, n} F_{m, n} u^{m} v^{n}$ do not evidently contribute. The reliability of the approximation (24) implies the series for the function $F(A, B)$ to be truncated at $m=A$ and $n=B$. Leaving more terms does not improve, but may worsen, the accuracy. Of course, it does not apply to the optical (25) or rigid target (26) approximations, where all the extra terms are already dropped out.

Representing the amplitude (7) as a power series in the parameter $g$ enables one to pick up the individual contribution of $n$-fold interaction as a coefficient in front of the $g^{n}$ term. They are large when taken separately, but due to the opposite signs they almost cancel each other, returning a final sum much smaller than any of them. Thus, the treatment of the amplitude in terms of the number of interactions seems to be quite unreasonable.

\section{HALO NUCLEI SCATTERING}

An interesting topic to apply our method to is the scattering of halo nuclei. The distinguishing feature of these nuclei is their large size exceeding that of the stable nuclei. They are assumed to be a composite system of a core and a halo [19,20], the density distribution being the sum of the two components [21-24],

$$
\rho(r)=N_{c} \rho_{c}(r)+N_{v} \rho_{v}(r) .
$$

The first and the second terms stand here for the core and for the halo densities, $N_{c}$ is the number of the nucleons in the core, and $N_{v}$ is the number of the valence neutrons in the halo [25]. 
TABLE II. The reaction cross sections in $\mathrm{mb}$ for ${ }^{11} \mathrm{Li}^{-12} \mathrm{C},{ }^{11} \mathrm{Be}-$ ${ }^{12} \mathrm{C}$, and ${ }^{14} \mathrm{Be}-{ }^{12} \mathrm{C}$ collisions at the energy $790 \mathrm{MeV}$ per nucleon. The first three columns are for the three density parametrizations (29), and the fourth column is for the "nonhalo" distributions. The experimental points in the fifth column are taken from Ref. [17].

\begin{tabular}{lrrrcc}
\hline \hline & \multicolumn{1}{c}{$G$} & \multicolumn{1}{c}{$O$} & \multicolumn{1}{c}{$2 S$} & "Nonhalo" & Expt. \\
\hline${ }^{11} \mathrm{Li}$ & 1024 & 1031 & 1033 & 1021 & $1040 \pm 60$ \\
${ }^{11} \mathrm{Be}$ & 911 & 918 & 916 & 914 & $942 \pm 8$ \\
${ }^{14} \mathrm{Be}$ & 1120 & 1128 & 1131 & 1103 & $1139 \pm 90$ \\
\hline \hline
\end{tabular}

Both the densities are taken in the Gaussian form, and the core density reads

$$
\rho_{c}(r)=\frac{1}{\pi^{\frac{3}{2}} a_{c}^{3}} e^{-\frac{r^{2}}{a_{c}^{2}}}, \quad a_{c}=\sqrt{2 / 3} R_{c},
$$

where $R_{c}$ is the core mean square radius, while the second part, $\rho_{v}$, usually admits three different parametrizations depending on the shell state the halo neutrons are supposed to occupy [25]:

$$
\begin{aligned}
\rho_{v}^{G}(r) & =\frac{1}{\pi^{\frac{3}{2}} a_{G}^{3}} e^{-\frac{r^{2}}{a_{G}^{2}}}, \quad \rho_{v}^{O}(r)=\frac{2}{3 \pi^{\frac{3}{2}} a_{O}^{5}} r^{2} e^{-\frac{r^{2}}{a_{O}^{2}}}, \\
\rho_{v}^{2 S}(r) & =\frac{2}{3 \pi^{\frac{3}{2}} a_{2 S}^{3}}\left(\frac{r^{2}}{a_{2 S}^{2}}-\frac{3}{2}\right)^{2} e^{-\frac{r^{2}}{a_{2 S}^{2}}}, \\
a_{G} & =\sqrt{2 / 3} R_{v}, \quad a_{O}=\sqrt{2 / 5} R_{v}, \quad a_{2 S}=\sqrt{2 / 7} R_{v},
\end{aligned}
$$

where $R_{v}$ is the halo mean square radius. There exists also a "nonhalo" distribution that neglects the halo and uses the density (27) fitted to the experimental matter radius [23].

In Table II we present the reaction cross sections for ${ }^{11} \mathrm{Li}$ ${ }^{12} \mathrm{C},{ }^{11} \mathrm{Be}-{ }^{12} \mathrm{C}$, and ${ }^{14} \mathrm{Be}-{ }^{12} \mathrm{C}$ scattering at the energy $790 \mathrm{MeV}$ per nucleon obtained through the exactly differentiated full generating function. The calculations have been carried out with the density (28) (normalized to unity) for all three parametrizations with [25]

$$
\begin{aligned}
& R_{c}=2.50 \mathrm{fm}, R_{v}=5.04 \mathrm{fm}, N_{c}=9, N_{v}=2 \text { for }{ }^{11} \mathrm{Li}, \\
& R_{c}=2.30 \mathrm{fm}, R_{v}=5.39 \mathrm{fm}, N_{c}=10, N_{v}=1 \text { for }{ }^{11} \mathrm{Be}, \\
& R_{c}=2.59 \mathrm{fm}, R_{v}=5.45 \mathrm{fm}, N_{c}=12, N_{v}=2 \text { for }{ }^{14} \mathrm{Be} .
\end{aligned}
$$

Besides we add the results for the "nonhalo" distributions with $R_{\text {rms }}=3.12$ fm for ${ }^{11} \mathrm{Li}, R_{\text {rms }}=2.73 \mathrm{fm}$ for ${ }^{11} \mathrm{Be}$, and $R_{\mathrm{rms}}=3.16 \mathrm{fm}$ for ${ }^{14} \mathrm{Be}$ [17]. The parametrization of ${ }^{12} \mathrm{C}$ is the same as in Table I.
The results for ${ }^{11} \mathrm{Li}$ and ${ }^{14} \mathrm{Be}$ are in a quite good agreement with the experimental data especially regarding the relatively large error bars. The evaluated cross sections for the ${ }^{11} \mathrm{Be}$ beam are systematically smaller although the deviation is not large. One might doubt whether the parameters are determined for this case with the proper accuracy. On the other hand the reason for the discrepancy could be in the nucleons' correlations.

It is worth pointing out that it is the reaction cross sections that have been calculated here, whereas the experimentally measured value is the interaction cross section. It is smaller than the reaction one, but their difference does not exceed $1-2 \%$ [18].

\section{CONCLUSION}

In this article we set up a novel approach to deal with Glauber amplitudes for nucleus-nucleus scattering at energies higher than several hundreds of $\mathrm{MeV}$ per nucleon. It is based on the closed expression obtained for the generating function. The main advantage it has is in a relatively simple analytical form that allows one to carry out calculations avoiding the complexities encountered in the Monte Carlo technique. As an example we apply our method to ${ }^{12} \mathrm{C}-{ }^{12} \mathrm{C}$ scattering at the energy $950 \mathrm{MeV}$ per nucleon for which there exist the experimental data [17]. We have calculated the reaction and the total cross sections for the mean square nuclear radius $R_{\text {rms }}=2.49 \mathrm{fm}$, the value taken from the Monte Carlo analysis in the harmonic oscillator parametrization in Ref. [13]. Our results are in good agreement with those obtained in that paper. As another example we have calculated the cross section of several halo nuclei scattering on a ${ }^{12} \mathrm{C}$ target at the energy $790 \mathrm{Mev}$ per nucleon.

The proposed generating function (20) is appropriate for any pairs of colliding nuclei regardless their atomic weight. Apart from the above-considered integrated cross sections, it can provide a consistent evaluation of the differential elastic cross section (1) as well. In this case, however, one has to account for the Coulomb corrections at small scattering angles for heavy nuclei.

Taking the nucleon density as the product of single-particle ones (8), we thereby neglect the nucleon-nucleon correlations. The particular correlations can be, in principle, accounted for in our approach, provided an appropriate wave function is known.

\section{ACKNOWLEDGMENTS}

We are grateful to I. S. Novikov for helpful discussions.
[1] I. Tanihata, H. Hamagaki, O. Hashimoto, S. Nagamiya, Y. Shida, N. Yoshikawa, O. Yamakawa, K. Sugimoto, T. Kobayashi, and D. E. Greiner et al., Measurements of interaction cross-sections and radii of He isotopes, Phys. Lett. B $\mathbf{1 6 0}$, 380 (1985).

[2] I. Tanihata, H. Hamagaki, O. Hashimoto, Y. Shida, N. Yoshikawa, K. Sugimoto, O. Yamakawa, T. Kobayashi, and N. Takahashi, Measurements of Interaction Cross-Sections and
Nuclear Radii in the Light $p$-Shell Region, Phys. Rev. Lett. 55, 2676 (1985).

[3] M. Smith, M. Brodeur, T. Brunner, S. Ettenauer, A. Lapierre, R. Ringle, V. L. Ryjkov, F. Ames, P. Bricault, G. W. F. Drake et al., First Penning-Trap Mass Measurement in the Millisecond Half-Life Range: The Exotic Halo Nucleus Li-11, Phys. Rev. Lett. 101, 202501 (2008).

[4] C. A. Bertulani, Nuclear reactions, arXiv:0908.3275. 
[5] R. J. Glauber, Cross-sections in deuterium at high-energies, Phys. Rev. 100, 242 (1955).

[6] R. J. Glauber and G. Matthiae, High-energy scattering of protons by nuclei, Nucl. Phys. B 21, 135 (1970).

[7] W. Czyz and L. C. Maximon, High-energy, small angle elastic scattering of strongly interacting composite particles, Ann. Phys. 52, 59 (1969).

[8] A. Bialas, M. Bleszynski, and W. Czyz, Relation between the Glauber model and classical probability calculus, Acta Phys. Pol., B 8, 389 (1977).

[9] G. D. Alkhazov, T. Bauer, R. Bertini, L. Bimbot, O. Bing, A. Boudard, G. Bruge, H. Catz, A. Chaumeaux, P. Couvert et al., Elastic and inelastic scattering of $1.37-\mathrm{GeV} \alpha$ particles from 40,42,44,48 Ca, Nucl. Phys. A 280, 365 (1977).

[10] A. M. Zadorozhnyi, V. V. Uzhinsky, and S. Y. Shmakov, A stochastic method of calculating nucleus-nucleus scattering characteristics in the eikonal approach, Sov. J. Nucl. Phys. 39, 729 JINR-P2-83-544 (1984).

[11] F. A. Gareev et al., Study of properties of exotic nuclei using elastic scattering. Theoretical consideration, Yad. Fiz. 58, 620 (1995); Phys. At. Nucl. 58, 564 (1995). .

[12] G. D. Alkhazov and A. A. Lobodenko, Reaction cross sections for collisions involving exotic light nuclei within the glauber approach, Yad. Fiz. 70, 98 (2007); Phys. At. Nucl. 70, 93 (2007).

[13] C. Merino, I. S. Novikov, and Y. M. Shabelski, Nuclear radii calculations in various theoretical approaches for nucleusnucleus interactions, Phys. Rev. C 80, 064616 (2009).

[14] C. Pajares and A. V. Ramallo, Effects of the multiple scattering structure in the propagation of hadronic properties in nucleusnucleus collisions, Phys. Rev. D 31, 2800 (1985).

[15] V. M. Braun and Y. M. Shabelski, Multiple scattering theory for inelastic processes, Int. J. Mod. Phys. A 3, 2417 (1988).

[16] K. G. Boreskov and A. B. Kaidalov, Nucleus-nucleus scattering in the Glauber approach, Sov. J. Nucl. Phys. 48, 367 (1988).
[17] A. Ozawa, T. Suzuki, and I. Tanihata, Nuclear size and related topics, Nucl. Phys. A 693, 32 (2001).

[18] I. S. Novikov and Y. Shabelski, Complete Glauber calculations of reaction and interaction cross sections for light-ion collisions, Phys. At. Nucl. 78, 951 (2015).

[19] I. Tanihata, Neutron halo nuclei, J. Phys. G: Nucl. Part. Phys. 22, 157 (1996).

[20] S. N. Ershov, T. Rogde, B. V. Danilin, J. S. Vaagen, I. J. Thompson, and F. A. Gareev, Halo excitation of ${ }^{6} \mathrm{He}$ in inelastic and charge exchange reactions, Phys. Rev. C 56, 1483 (1997).

[21] G. D. Alkhazov, A. V. Dobrovolsky, and A. A. Lobodenko, Matter density distributions and radii of light exotic nuclei from intermediate-energy proton elastic scattering and from interaction cross sections, Nucl. Phys. A 734, 361 (2004).

[22] S. Ilieva, F. Aksouh, G. D. Alkhazov, L. Chulkov, A. V. Dobrovolsky, P. Egelhof, H. Geissel, M. Gorska, A. Inglessi, R. Kanungo et al., Nuclear-matter density distribution in the neutron-rich nuclei $12,14 \mathrm{Be}$ from proton elastic scattering in inverse kinematics, Nucl. Phys. A 875, 8 (2012).

[23] A. A. Korsheninnikov, E. Y. Nikolskii, C. A. Bertulani, S. Fukuda, T. Kobayashi, E. A. Kuzmin, S. Momota, B. G. Novatskii, A. A. Ogloblin, A. Ozawa et al., Scattering of radioactive nuclei $6 \mathrm{He}$ and $3 \mathrm{H}$ by protons: Effects of neutron skin and halo in $6 \mathrm{He}, 8 \mathrm{He}$, and $11 \mathrm{Li}$, Nucl. Phys. A 617, 45 (1997).

[24] M. V. Zhukov, B. V. Danilin, D. V. Fedorov, J. M. Bang, I. J. Thompson, and J. S. Vaagen, Bound state properties of Borromean Halo nuclei: ${ }^{6} \mathrm{He}$ and ${ }^{11} \mathrm{Li}$, Phys. Rep. 231, 151 (1993).

[25] M. A. M. Hassan, M. S. M. Nour El-Din, A. Ellithi, E. Ismail, and H. Hosny, The effect of halo nuclear density on reaction cross-section for light ion collision, Int. J. Mod. Phys. E 24, 1550062 (2015). 